03

\title{
Детально-сравнительный анализ взаимодействия сверхзвукового потока с поперечной газовой струей при больших параметрах нерасчетности
}

\author{
(C) A.О. Бекетаева, ${ }^{1}$ P. Bruel, ${ }^{2}$ А.Ж. Найманова ${ }^{3}$ \\ ${ }^{1}$ Казахский национальный университет им. Аль-Фараби, \\ 050040 Алматы, Казахстан \\ ${ }^{2}$ Национальный центр научных исследований, \\ По, Франция \\ ${ }^{3}$ Институт математики и математического моделирования Министерства образования и науки Республики Казахстан, \\ 050010 Алматы, Казахстан \\ e-mail: azimaras10@gmail.com, azimaras@mail.ru
}

Поступило в Редакцию 17 января 2019 г.

В окончательной редакции 17 января 2019 г.

Принято к публикации 24 апреля 2019 г.

\begin{abstract}
Взаимодействие пространственного сверхзвукового турбулентного потока газа с вдуваемой перпендикулярно со стенки звуковой струей было широко изучено как численно, так и экспериментально. Однако основным недостатком этих исследований является отсутствие детализации образования и распространения вихревых структур, как для умеренных, так и больших параметров нерасчетности $n$ (отношение давления в струе к давлению в потоке). Выполненное в данной работе исследование направлено на выявление и углубленное понимание механизмов образования вихрей за вдуваемой звуковой струи в сверхзвуковом натекающем потоке в зависимости от $n$ с точки зрения повышения эффективности перемешивания струи и потока. Исходными являются трехмерные осредненные по Фавру уравнения Навье-Стокса, замкнутые $k-\omega$ моделью турбулентности, решение которых осуществляется с помощью алгоритма, построенного на основе ENO-схемы третьего порядка аппроксимации. Показано присутствие известных из ряда теоретических работ вихревых структур: два противоположно вращающихся вихря перед струей; подковообразный вихрь; две пары, формирующиеся в зоне смешения между струей и потоком, одна из которых в следе за струей, одна по боковой линии струи. Определены параметры нерасчетности, при которых появляются дополнительные пары вихрей, где одна пара возникает на кромке диска Маха в результате взаимодействия замедленного потока струи за диском Маха с высокоскоростным восходящим потоком за бочкой, а вторая обусловлена взаимодействием восходящего потока струи с натекающим основным потоком газа. В результате сравнительного анализа выявлены параметры нерасчетности, при которых наблюдается четкая картина дополнительных роговых вихрей вблизи стенки в области за струей. Получен график зависимости угла наклона головного скачка уплотнения от параметра нерасчетности. Установлено удовлетворительное согласие распределения давления на стенке перед струей в плоскости симметрии с экспериментальными данными.
\end{abstract}

Ключевые слова: численное моделирование, сверхзвуковое течение, совершенный газ, пограничный слой, уравнения Навье-Стокса, параметр нерасчетности, ударная волна.

DOI: 10.21883/JTF.2019.10.48166.12-19

\section{Введение}

Течения высокоскоростных струй в поперечном потоке обеспечивают эффективное смешивание топлива и окислителя, имеющего решающее значение для сверхзвукового горения. На данный момент известно большое количество экспериментальных [1-5] и теоретических [6-14] исследований, в которых достаточно хорошо изучен механизм образования ударно-волновой структуры струйного взаимодействия для умеренных параметров нерасчетности $n$ (отношение давления в струе к давлению в потоке). Общую картину течения схематически можно представить следующим образом (рис. 1, $a$ пространственная картина, $b-$ на линии симметрии): линия 1 - головной скачок уплотнения, возникающий вследствие торможения набегающего потока перед струей, линии 2,3 - косой и замыкающий скачки уплот- нения соответственно. Головной, косой и замыкающий скачки уплотнения, пересекаясь в одной точке, образуют $\lambda$-образную структуру. Буквами $D$ и $B$ обозначены диск Маха и бочкообразная структура в струе соответственно. Линиями 4 и 5 изображены хорошо известные два противоположно вращающихся вихря перед струей, появляющихся из-за большого градиента давления, создаваемого на стенке перед вдуваемой струей и, как следствие, отрыва пограничного слоя. На рис. 1,c схематически иллюстрируется вихревая структура за струей, которая, как известно, вносит существенный вклад в смешение топлива и окислителя. Здесь линией 6 представлен вихревой след, 8 - это общеизвестная противоположновращающаяся пара вихрей в самой струе, образованная прогибом струи и конвекцией поперечного потока, также здесь приведен подковообразный вихрь 7. 
Однако в работе [9] в результате численного моделирования течения с параметром нерасчетности $n=282$ и в расчетах [13], проведенных с параметрами нерасчетности в диапазоне $10 \leq n \leq 50$, выявлено наличие дополнительных пар вихрей за струей, которая схематически также иллюстрируется на рис. 1, c. В этой картине новыми являются вихревые структуры 9 и 10, где пара вихрей 9 возникает за счет взаимодействия струи, проходящей через диск Маха с восходящим внешним потоком, а вихри 10 зарождаются за счет взаимодействия потока с бочкообразной структурой в струе.

Следует также отметить, что исследования авторов [10] для параметра нерасчетности $5 \leq n \leq 2000$ подтверждают наличие известных вихрей и показывают дополнительную пару вихрей (роговых) за вдуваемой струей непосредственно возле стенки, где пограничный слой обтекает струю, возникающую как в дозвуковом, так и в сверхзвуковом течениях, однако в работах $[9,13]$ эти вихри не были выявлены. Представляется затруднительным проведение сравнительного анализа полученных данных с результатами работы [10], так как в этой работе не приведено количественное описание возникновения и распространения вихрей. Все это свидетельствует о необходимости дальнейшего исследования вихревых структур в широком диапазоне параметра нерасчетности.

Целью исследования настоящей работы является численное изучение и детально-сравнительный анализ механизмов формирования вихревых и ударно-волновых структур в широком диапазоне параметра нерасчетности, а также получение обобщенных зависимостей характеристик потока в зависимости от этого параметра. Для этого численно моделируется задача вдува звуковых струй из круглых отверстий, расположенных симметрично на верхней и нижней стенках канала, перпендикулярно сверхзвуковому потоку. Для удобства вычисления рассматривается вдув струи только с нижней стенки. Схема течения и качественная картина вихревой структуры показаны на рис. 1.

\section{Постановка задачи}

Исходной является система трехмерных осредненных по Фавру уравнений Навье-Стокса для сжимаемого турбулентного газа, записанная в декартовой системе координат в консервативной форме

$$
\frac{\partial \mathbf{U}}{\partial t}+\frac{\partial\left(\mathbf{E}-\mathbf{E}_{v}\right)}{\partial x}+\frac{\partial\left(\mathbf{F}-\mathbf{F}_{v}\right)}{\partial z}+\frac{\partial\left(\mathbf{G}-\mathbf{G}_{v}\right)}{\partial y}=\mathbf{S},
$$

компоненты векторов $\mathbf{U}, \mathbf{E}, \mathbf{F}, \mathbf{G}$ определяются выражениями

$$
\mathbf{U}=\left(\begin{array}{c}
\rho \\
\rho u \\
\rho v \\
\rho w \\
E_{t} \\
\rho k \\
\rho \omega
\end{array}\right), \quad \mathbf{E}=\left(\begin{array}{c}
\rho u \\
\rho u^{2}+P \\
\rho u v \\
\rho u w \\
\left(E_{t}+P\right) u \\
\rho u k \\
\rho u \omega
\end{array}\right)
$$

$$
\mathbf{F}=\left(\begin{array}{c}
\rho w \\
\rho u w \\
\rho v w \\
\rho w^{2}+P \\
\left(E_{t}+P\right) w \\
\rho w k \\
\rho w \omega
\end{array}\right), \quad \mathbf{G}=\left(\begin{array}{c}
\rho v \\
\rho u v \\
\rho v^{2}+P \\
\rho v w \\
\left(E_{t}+P\right) v \\
\rho v k \\
\rho v \omega
\end{array}\right)
$$

а компоненты $\mathbf{E}_{v}, \mathbf{F}_{v}, \mathbf{G}_{v}$ связаны с вязкими напряжениями

$$
\begin{aligned}
\mathbf{E}_{v}= & \left(0, \tau_{x x}, \tau_{x y}, \tau_{x z}, u \tau_{x x}+v \tau_{x z}+w \tau_{x z}-q_{x},\right. \\
& \left.\frac{1}{\operatorname{Re}}\left(\mu_{l}+\sigma_{k} \mu_{t}\right) \frac{\partial k}{\partial x}, \frac{1}{\operatorname{Re}}\left(\mu_{l}+\sigma_{\omega} \mu\right) \frac{\partial \omega}{\partial x}\right)^{T}, \\
\mathbf{F}_{v}= & \left(0, \tau_{x z}, \tau_{y z}, \tau_{z z}, u \tau_{x z}+v \tau_{y z}+w \tau_{z z}-q_{z},\right. \\
& \left.\frac{1}{\operatorname{Re}}\left(\mu_{l}+\sigma_{k} \mu_{t}\right) \frac{\partial k}{\partial z}, \frac{1}{\operatorname{Re}}\left(\mu_{l}+\sigma_{\omega} \mu\right) \frac{\partial \omega}{\partial z}\right)^{T}, \\
\mathbf{G}_{v}= & \left(0, \tau_{x y}, \tau_{y y}, \tau_{y z}, u \tau_{y y}+v \tau_{y y}+w \tau_{y z}-q_{y},\right. \\
& \left.\frac{1}{\operatorname{Re}}\left(\mu_{l}+\sigma_{k} \mu_{t}\right) \frac{\partial k}{\partial y}, \frac{1}{\operatorname{Re}}\left(\mu_{l}+\sigma_{\omega} \mu\right) \frac{\partial \omega}{\partial y}\right)^{T} .
\end{aligned}
$$

Тензоры напряжения и потоки тепла выражаются в виде

$$
\begin{gathered}
\tau_{x x}=\frac{2}{3} \frac{\mu}{\operatorname{Re}}\left(2 \frac{\partial u}{\partial x}-\frac{\partial w}{\partial z}-\frac{\partial v}{\partial y}\right), \\
\tau_{z z}=\frac{2}{3} \frac{\mu}{\operatorname{Re}}\left(2 \frac{\partial w}{\partial z}-\frac{\partial u}{\partial x}-\frac{\partial v}{\partial y}\right), \\
\tau_{y y}=\frac{2}{3} \frac{\mu}{\operatorname{Re}}\left(2 \frac{\partial v}{\partial y}-\frac{\partial u}{\partial x}-\frac{\partial w}{\partial z}\right), \\
\tau_{x z}=\tau_{z x}=\frac{\mu}{\operatorname{Re}}\left(\frac{\partial u}{\partial z}+\frac{\partial w}{\partial x}\right), \\
\tau_{x y}=\tau_{y x}=\frac{\mu}{\operatorname{Re}}\left(\frac{\partial u}{\partial y}+\frac{\partial v}{\partial x}\right), \\
\tau_{y z}=\tau_{z y}=\frac{\mu}{\operatorname{Re}}\left(\frac{\partial w}{\partial x}+\frac{\partial v}{\partial z}\right), \\
q_{x}=\frac{\mu}{(\gamma-1) M_{\infty}^{2} \operatorname{Pr} \operatorname{Re}} \frac{\partial T}{\partial x}, \\
q_{y}=\frac{\mu}{(\gamma-1) M_{\infty}^{2} \operatorname{Pr} \operatorname{Re}} \frac{\partial T}{\partial y}, \\
q_{z}=\frac{\mu}{(\gamma-1) M_{\infty}^{2} \operatorname{Pr} \operatorname{Re}} \frac{\partial T}{\partial z} .
\end{gathered}
$$

Здесь $\mu=\mu_{l}+\mu_{t}$, где $\mu_{l}-$ молекулярная вязкость (определяется по формуле Сазерленда), $\mu_{t}$ - турбулентная вязкость.

Вектор дополнительных членов имеет следующий вид:

$\mathbf{S}=\left(0,0,0,0,0,\left(P_{k}-\beta^{*} \rho \omega k\right),\left(\gamma^{*} \rho P_{k} / \mu_{t}-\beta \rho \omega^{2}\right)\right)^{T}$, 

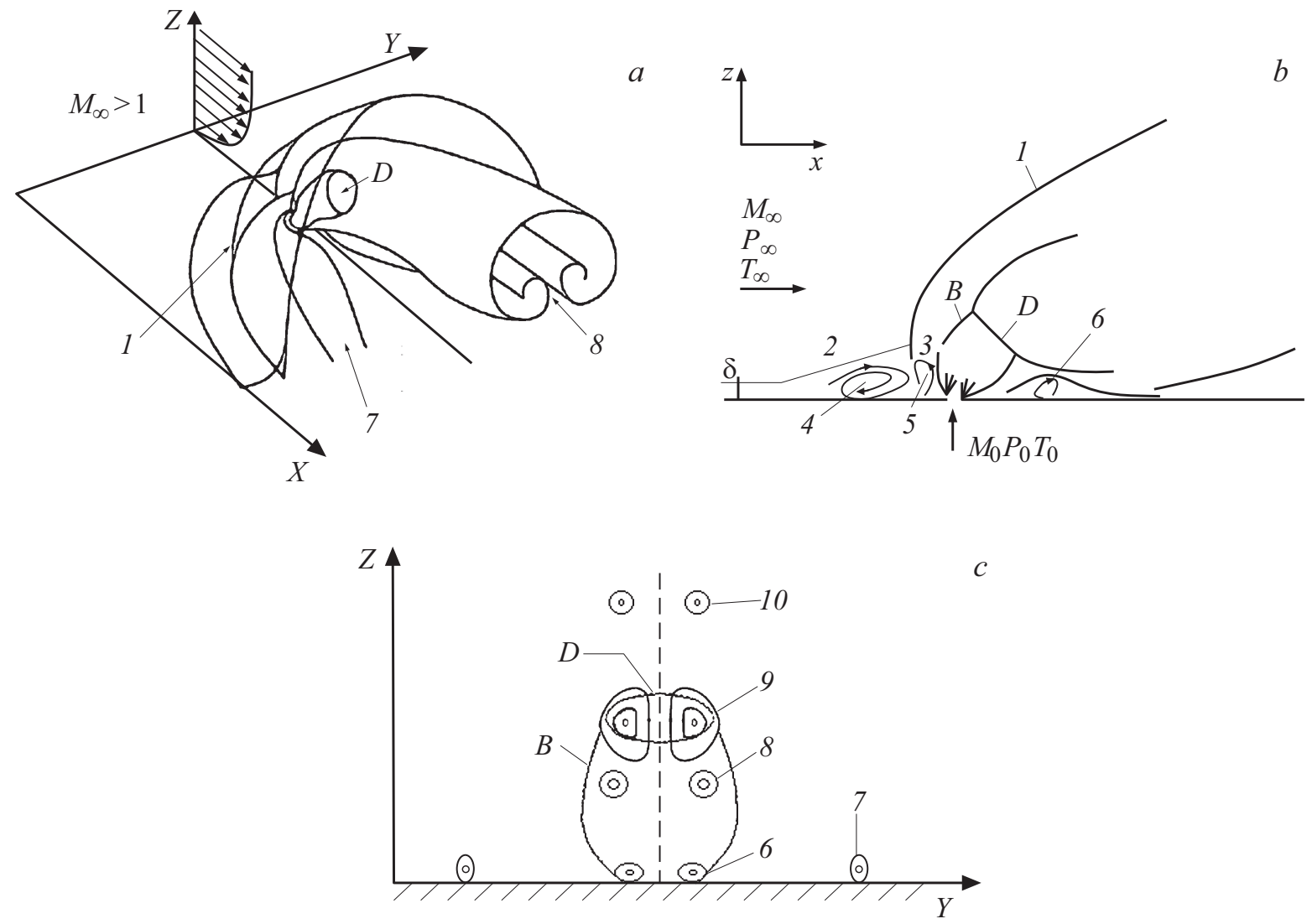

Рис. 1. Схема течения. Пояснения в тексте.

где

$$
\begin{gathered}
P_{k}=\mu_{t}\left[\left(\frac{\partial u_{i}}{\partial x_{j}}+\frac{\partial u_{j}}{\partial x_{i}}\right) \frac{\partial u_{i}}{\partial x_{j}}-\frac{2}{3}\left(\frac{\partial u_{k}}{\partial x_{k}}\right)^{2}\right]-\frac{2}{3} \rho k \frac{\partial u_{k}}{\partial x_{k}}, \\
i, j, k=1,2,3
\end{gathered}
$$

Константы принимают следующие значения:

$$
\sigma_{k}=0.5, \sigma_{\omega}=0.5, \beta^{*}=0.09, \beta=0.075, \gamma^{*}=5 / 9 .
$$

Здесь $k, \omega-$ кинетическая энергия турбулентности и скорость диссипации кинетической энергии турбулентности, $P_{k}$ - член генерации турбулентности, турбулентная вязкость определяется по формуле $\mu_{t}=\frac{\rho k}{\omega}[15]$.

Выражения для давления и температуры запишутся следующим образом:

$$
\begin{gathered}
P=(\gamma-1)\left[E_{t}-\frac{1}{2}\left(\rho u^{2}+\rho w^{2}+\rho v^{2}\right)\right], \\
T=\left(\frac{1}{\rho c_{v}}\right)\left[E_{t}-\frac{1}{2}\left(\rho u^{2}+\rho w^{2}+\rho v^{2}\right)\right], \\
c_{v}=\frac{1}{\gamma(\gamma-1) M_{\infty}^{2}} .
\end{gathered}
$$

Здесь $t-$ время, $u, w, v-$ компоненты скорости потока в продольном и поперечных направлениях, $\rho-$ плотность, $E_{t}-$ полная энергия, $P$ - давление, $T-$ температура, $c_{v}$ - теплоемкость при постоянном объеме, $\gamma$ - показатель адиабаты, $M_{\infty}$ - число Маха потока, далее индекс 0 отнесен к параметрам струи, $\infty$ - к параметрам потока.

Исходная система (1) записана в безразмерной форме. В качестве определяющих параметров приняты размерные параметры на входе $\hat{u}_{\infty}, \hat{\rho}_{\infty}, \hat{T}_{\infty}$, давление и полная энергия отнесены к значению $\hat{\rho}_{\infty} \hat{u}_{\infty}^{2}$, характерным размером длины является диаметр круглого отверстия струи $\hat{d}$. $\operatorname{Pr}-$ число Прандля, $\operatorname{Re}=\hat{u}_{\infty} \hat{\rho}_{\infty} \hat{L} / \mu-$ число Рейнольдса, где $\hat{L}$ - длина канала, являющаяся определяющим размером для Re.

Граничные и начальные условия имеют следующий вид: на входе, а также в качестве начальных данных задаются параметры потока

$$
\begin{gathered}
u=1, \quad v=0, \quad w=0, \quad \rho=1, \quad T_{\infty}=1, \\
x=0, \quad 0 \leq y \leq H_{y}, \quad 0 \leq z \leq H_{z},
\end{gathered}
$$

Начальные данные для $k, \omega$ параметров определялись с использованием алгебраической модели турбулентности Болдуина-Ломакса по известным осредненным физическим параметрам входного потока и подробно приведены в работе [13].

Вблизи стенки задается пограничный слой, толщина которого определяется по формуле $\delta_{1}=0.37 x(\mathrm{Re})^{-0.2}$. Также задается пристенный слой $(10 \%$ от пограничного 
слоя) $\delta_{2}=0.1 \delta_{1}$. Продольная составляющая скорости $u$, а также температура и плотности аппроксимируются в соответствии с работой [13]:

на струе:

$$
\begin{gathered}
u=0, \quad v=0, \quad w=\sqrt{T_{0}} M_{0} / M_{\infty}, \quad P_{0}=n P_{\infty}, \\
T_{0}=3.71, \quad z=0, \quad\left|x^{2}+y^{2}\right| \leq R .
\end{gathered}
$$

Здесь $n=P_{0} / P_{\infty}$ - параметр нерасчетности.

на верхней границе задается условие симметрии:

$$
\begin{gathered}
w=0 ; \quad \frac{\partial u}{\partial z}=0 ; \quad \frac{\partial v}{\partial z}=0 ; \quad \frac{\partial T}{\partial z}=0 ; \quad \frac{\partial k}{\partial z}=0 ; \quad \frac{\partial \omega}{\partial z}=0 ; \\
z=H_{z}, \quad 0 \leq x \leq H_{x}, \quad 0 \leq y \leq H_{y} ;
\end{gathered}
$$

на боковых границах:

$$
\begin{gathered}
\frac{\partial u}{\partial y}=\frac{\partial v}{\partial y}=\frac{\partial w}{\partial y}=\frac{\partial \rho}{\partial y}=\frac{\partial k}{\partial y}=\frac{\partial \omega}{\partial y}=0, \\
y=0, \quad y=H_{y}, \quad 0 \leq x \leq H_{x}, \quad 0 \leq z \leq H_{z},
\end{gathered}
$$

где $H_{x}=\hat{L} / \hat{d}$ - длина, $H_{z}$ - высота, $H_{y}$ - ширина безразмерной расчетной области, $R=0.5$ - радиус круглого отверстия; на выходной границе задается условие неотражения [16].

\section{Метод решения}

В работе $[17,18]$ изложена ENO-схема на основе идеи метода Годунова и продемонстрирована применимость схемы к решению задачи сверхзвукового течения многокомпонентного газа в плоском канале с вдувом перпендикулярных струй. В работе [13] разработанный алгоритм обобщается на трехмерный случай, и показана возможность проведения достоверных численных расчетов сложных сверхзвуковых пространственных течений. В соответствии с этим предварительно в пограничном слое вблизи стенки и на уровне струи для более точного учета течения, вводится сгущение сетки с помощью преобразований [18]:

$$
\xi=\xi(x), \quad \eta=\eta(z), \quad \xi=\xi(y) .
$$

При этом система уравнений (1) в обобщенных координатах запишется в виде

$$
\begin{aligned}
\frac{\partial \tilde{\mathbf{U}}}{\partial t}+\frac{\partial \tilde{\mathbf{E}}}{\partial \xi}+\frac{\partial \tilde{\mathbf{F}}}{\partial \eta} & +\frac{\partial \tilde{\mathbf{G}}}{\partial \xi}=\frac{\partial \tilde{\mathbf{E}}_{v 2}}{\partial \xi}+\frac{\partial \tilde{\mathbf{E}}_{v m}}{\partial \xi}+\frac{\partial \tilde{\mathbf{F}}_{v 2}}{\partial \eta} \\
& +\frac{\partial \tilde{\mathbf{F}}_{v m}}{\partial \eta}+\frac{\partial \tilde{\mathbf{G}}_{v 2}}{\partial \xi}+\frac{\partial \tilde{\mathbf{G}}_{v m}}{\partial \xi} .
\end{aligned}
$$

где

$$
\begin{gathered}
\tilde{\mathbf{U}}=\frac{1}{J} \mathbf{U}, \quad \tilde{\mathbf{E}}=\left(\frac{\xi_{x}}{J}\right) \mathbf{E}, \quad \tilde{\mathbf{F}}=\left(\frac{\eta_{z}}{J}\right) \mathbf{F}, \\
\tilde{\mathbf{E}}_{v 2}=\left(\frac{\xi_{x}}{J}\right) \mathbf{E}_{v 2}, \quad \tilde{\mathbf{E}}_{v m}=\left(\frac{\xi_{x}}{J}\right) \mathbf{E}_{v m}, \\
\tilde{\mathbf{F}}_{v 2}=\left(\frac{\eta_{z}}{J}\right) \mathbf{F}_{v 2}, \quad \tilde{\mathbf{F}}_{v m}=\left(\frac{\eta_{z}}{J}\right) \mathbf{F}_{v m},
\end{gathered}
$$

$$
\begin{gathered}
\tilde{\mathbf{G}}_{v 2}=\left(\frac{\xi_{y}}{J}\right) \mathbf{G}_{v 2}, \quad \tilde{\mathbf{G}}_{v m}=\left(\frac{\xi_{y}}{J}\right) \mathbf{G}_{v m}, \\
J=\frac{\partial(\xi, \eta, \xi)}{\partial(x, z, y)}
\end{gathered}
$$

- якобиан преобразования, $\tilde{\mathbf{E}}_{v m}, \tilde{\mathbf{E}}_{v 2}$ - диффузионные члены, содержащие смешанные и вторые производные.

В соответствии с принципом построения ENO-схемы, исходная система уравнений формально представляется следующим образом:

$$
\begin{gathered}
\frac{\partial \tilde{\mathbf{U}}}{\partial t}+\left(\hat{A}^{+}+\hat{A}^{-}\right) \frac{\partial \mathbf{E}^{m}}{\partial \xi}+\left(\hat{B}^{+}+\hat{B}^{-}\right) \frac{\partial \mathbf{F}^{m}}{\partial \eta}+\left(\hat{Q}^{+}+\hat{Q}^{-}\right) \frac{\partial \mathbf{G}^{m}}{\partial \xi} \\
-\left[\frac{\partial\left(\tilde{\mathbf{E}}_{v 2}+\tilde{\mathbf{E}}_{v m}\right)}{\partial \xi}+\frac{\partial\left(\tilde{\mathbf{F}}_{v 2}+\tilde{\mathbf{F}}_{v m}\right)}{\partial \eta}-\frac{\partial\left(\tilde{\mathbf{G}}_{v 2}+\tilde{\mathbf{G}}_{v m}\right)}{\partial \xi}\right]=0 .
\end{gathered}
$$

Здесь

- матрицы Якоби,

$$
A=\frac{\partial \mathbf{E}}{\partial \mathbf{U}}, \quad B=\frac{\partial \mathbf{F}}{\partial \mathbf{U}}, \quad Q=\frac{\partial \mathbf{G}}{\partial \mathbf{U}}
$$

$$
\begin{gathered}
\hat{A}^{ \pm}=R \hat{\Lambda}_{\xi} R^{-1}=R\left(\frac{1 \pm \operatorname{sign}\left(\Lambda_{\xi}\right)}{2}\right) R^{-1} . \\
\hat{B}^{ \pm}=T \hat{\Lambda}_{\eta} T^{-1}=T\left(\frac{1 \pm \operatorname{sign}\left(\Lambda_{\eta}\right)}{2}\right) T^{-1}, \\
\hat{Q}^{ \pm}=S \hat{\Lambda}_{\xi} S^{-1}=S\left(\frac{1 \pm \operatorname{sign}\left(\Lambda_{\zeta}\right)}{2}\right) S^{-1},
\end{gathered}
$$

$\mathbf{E}^{m}=\tilde{\mathbf{E}}+\mathbf{E}_{\xi}+\mathbf{D}_{\xi}, \mathbf{F}^{m}=\tilde{\mathbf{F}}+\mathbf{E}_{\eta}+\mathbf{D}_{\eta}, \mathbf{G}^{m}=\tilde{\mathbf{G}}+\mathbf{E}_{\xi}+\mathbf{D}_{\xi}$ модифицированные потоки в узловых точках $(i, j, k)$, состоящие из исходных конвективных векторов $(\tilde{\mathbf{E}}, \tilde{\mathbf{F}}, \tilde{\mathbf{G}})$ и добавочных членов высокого порядка точности $\left(\mathbf{E}_{\xi}, \mathbf{D}_{\xi}\right.$, $\left.\mathbf{E}_{\eta}, \mathbf{D}_{\eta}, \mathbf{E}_{\xi}, \mathbf{D}_{\xi}\right)$, подробно описанных в $[17,18]$.

После факторизации одношаговой конечноразностной схемы для интегрирования по времени уравнения (4) получается следующее равенство:

$$
\begin{aligned}
& \left\{I+\Delta t\left[\left(\hat{A}^{+}+\hat{A}^{-}\right)^{n} \frac{\partial}{\partial \xi} A_{\xi}^{n} \bullet-\frac{\partial}{\partial \xi} \tilde{\mu}_{\xi} \frac{\partial}{\partial \xi} \frac{1}{\tilde{\mathbf{U}}_{I}} \bullet\right]\right\} \\
& \times\left\{I+\Delta t\left[\left(\hat{B}^{+}+\hat{B}^{-}\right)^{n} \frac{\partial}{\partial \eta} B_{\eta}^{n} \bullet-\frac{\partial}{\partial \eta} \tilde{\mu}_{\eta} \frac{\partial}{\partial \eta} \frac{1}{\tilde{\mathbf{U}}_{I}} \bullet\right]\right\} \\
& \times\left\{I+\Delta t\left[\left(\hat{Q}^{+}+\hat{Q}^{-}\right)^{n} \frac{\partial}{\partial \xi} Q_{\xi}^{n} \bullet-\frac{\partial}{\partial \xi} \tilde{\mu}_{\xi} \frac{\partial}{\partial \xi} \frac{1}{\tilde{\mathbf{U}}_{I}} \bullet\right]\right\} \tilde{\mathbf{U}}^{n+1} \\
& =\tilde{\mathbf{U}}^{n}+\Delta t\left[\frac{\partial \tilde{\mathbf{E}}_{v 22}^{n}}{\partial \xi}+\frac{\partial \tilde{\mathbf{F}}_{v 22}^{n}}{\partial \eta}+\frac{\partial \tilde{\mathbf{G}}_{v 22}^{n}}{\partial \xi}+\frac{\partial}{\partial \xi}\left(2 \tilde{E}_{v m}^{n}-\tilde{\mathbf{E}}_{v m}^{n-1}\right)\right. \\
& \left.+\frac{\partial}{\partial \eta}\left(2 \tilde{F}_{v m}^{n}-\tilde{\mathbf{F}}_{v m}^{n-1}\right)+\frac{\partial}{\partial \xi}\left(2 \tilde{G}_{v m}^{n}-\tilde{\mathbf{G}}_{v m}^{n-1}\right)\right] \\
& -\Delta t\left[\left(\hat{A}^{+}+\hat{A}^{-}\right) \frac{\partial}{\partial \xi}\left(\mathbf{E}_{\xi}+\mathbf{D}_{\xi}\right)+\left(\hat{B}^{+}+\hat{B}^{-}\right) \frac{\partial}{\partial \eta}\left(\mathbf{E}_{\eta}+\mathbf{D}_{\eta}\right)\right. \\
& \left.\quad+\left(\hat{Q}^{+}+\hat{Q}^{-}\right) \frac{\partial}{\partial \xi}\left(\mathbf{E}_{\xi}+\mathbf{D}_{\xi}\right)\right]^{n} \cdot
\end{aligned}
$$

где $A_{\xi}=\xi_{x} A, B_{\eta}=\eta_{z} B, Q_{\xi}=\xi_{y} Q$ причем $\hat{A}^{+}+\hat{A}^{-}=I$, $I$ - единичная матрица,

$$
\tilde{\mu}_{\xi}=\frac{\mu \xi_{x}^{2}}{\operatorname{Re} J}, \quad \tilde{\mu}_{\eta}=\frac{\mu \eta_{z}^{2}}{\operatorname{Re} J}, \quad \tilde{\mu}_{\xi}=\frac{\mu \xi_{y}^{2}}{\operatorname{Re} J} .
$$



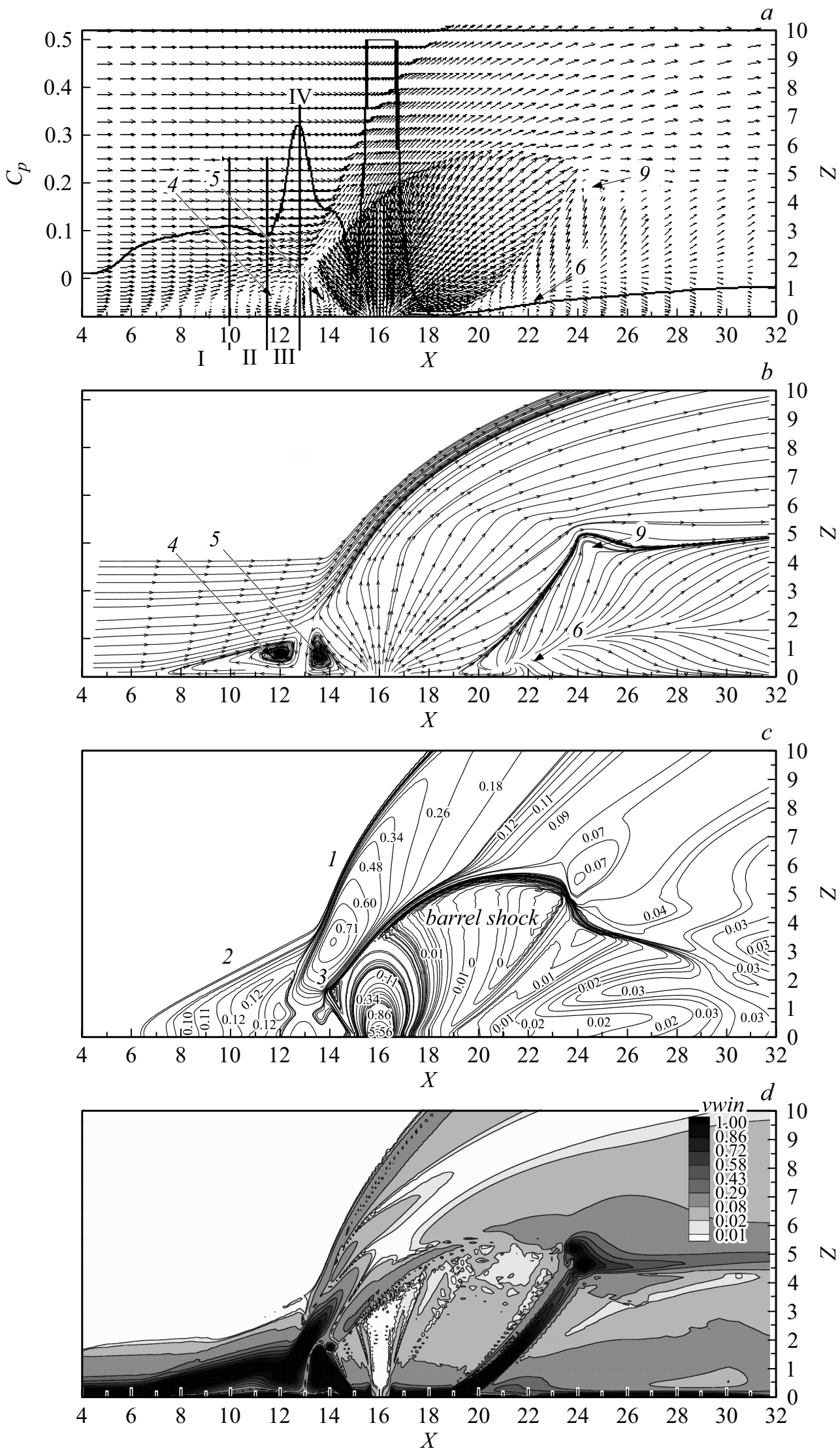

Pис. 2. $a$ - поле вектора скорости и коэффициент давления на стенке $C_{P} ; b-$ проекции линий тока на плоскость, нормальную к оси $x ; c-$ линии изобар; $d-$ величина завихренности, при $\operatorname{Re}=1.87 \cdot 10^{6}, \operatorname{Pr}=0.72, n=300, M_{0}=1, M_{\infty}=4$, в плоскости симметрии сечение $y=16$. 

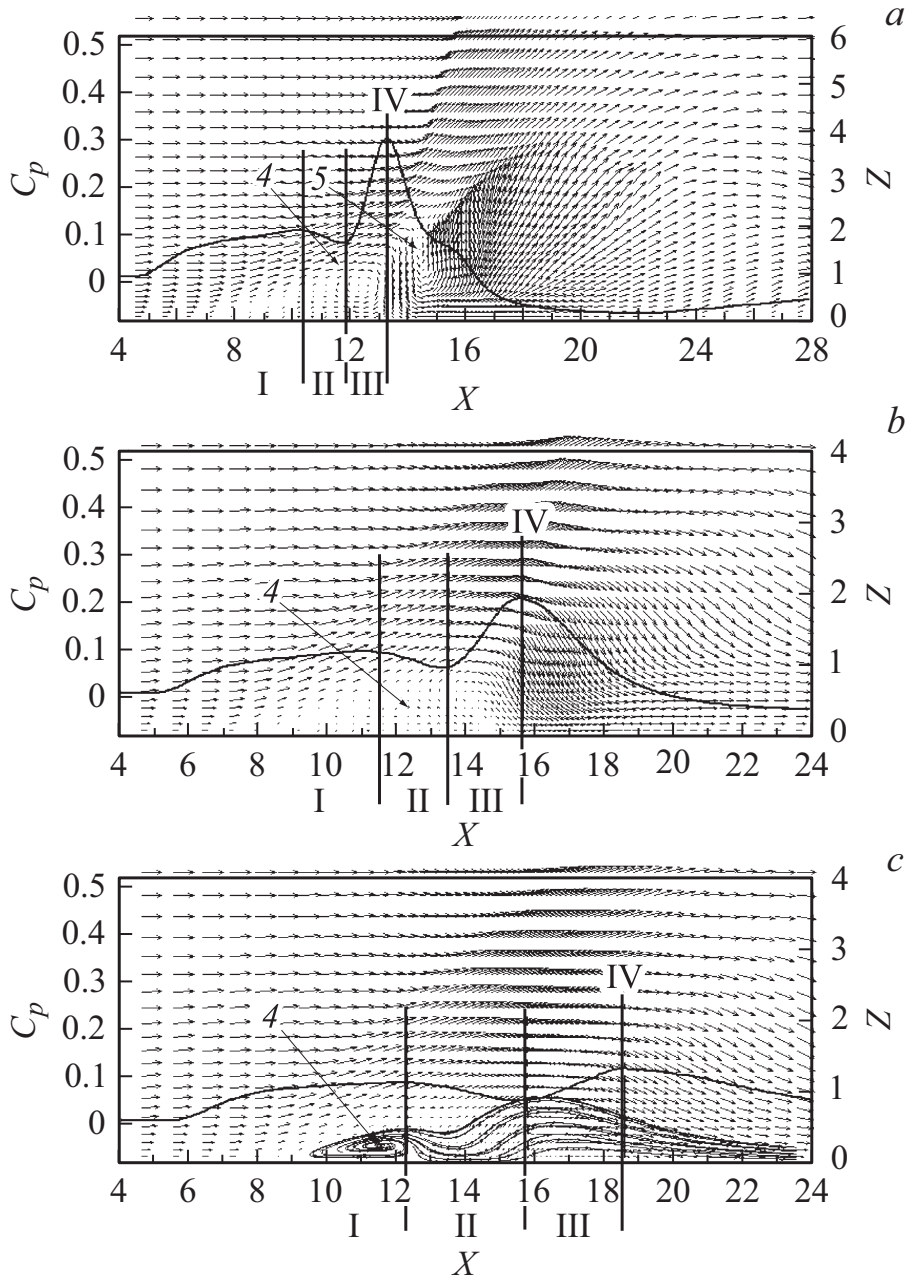

$a$

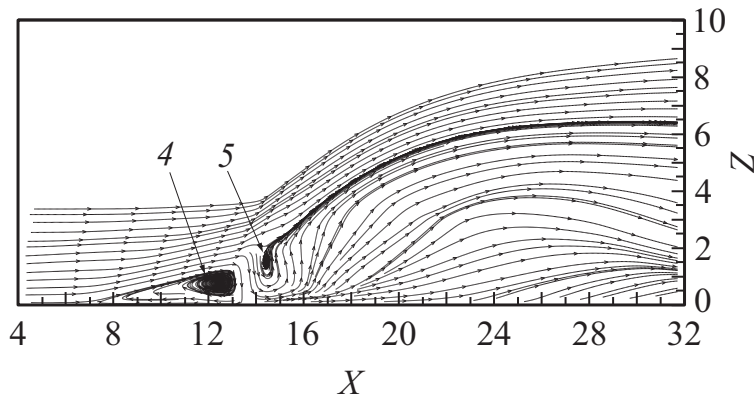

$b$
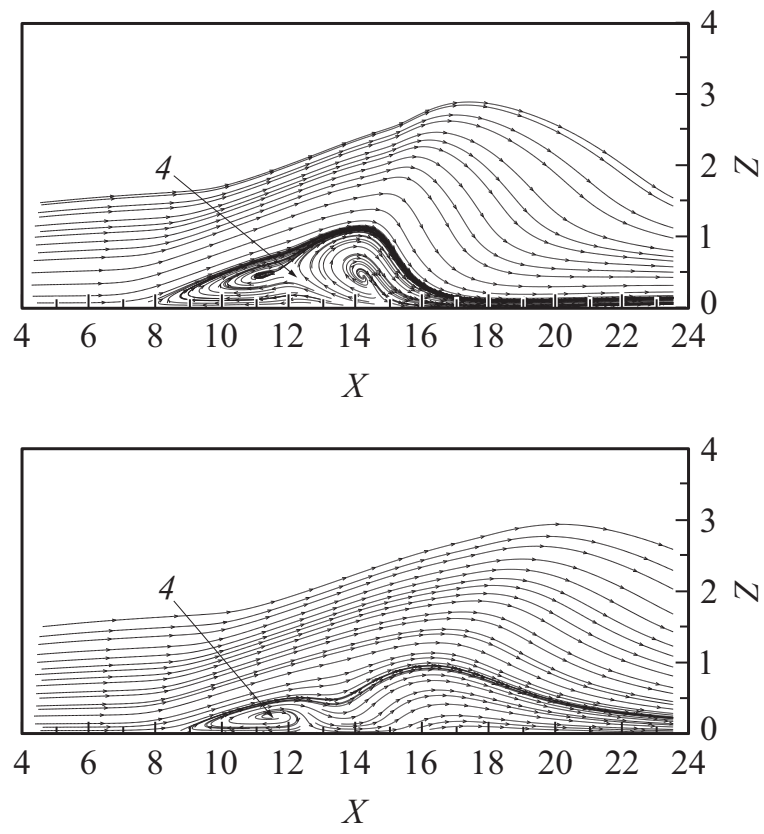

Рис. 3. Поле вектора скорости и коэффициент давления на стенке $C_{P}$ (слева), проекции линий тока на плоскость, нормальную к оси $y$ (справа) при $\operatorname{Re}=1.87 \cdot 10^{6}, \operatorname{Pr}=0.72, n=300, M_{0}=1, M_{\infty}=4$ в плоскости $z x: a-$ сечение $y=18.41, b-y=20.97$, $c-y=22.98$.

Аппроксимация производных в конвективных членах, членах содержащих добавочные вектора высокого поряда, а также в членах, содержащих вторые и смешанные производные, производится согласно $[17,18]$. Решение системы (5) осуществляется методом расщепления относительно вектора $\tilde{\mathbf{U}}$ матричной прогонкой.

\section{Анализ результатов}

Исследуется обтекание звуковой струи совершенного газа $\left(M_{0}=1\right)$ с диаметром отверстия $\hat{d}=0.476 \mathrm{~cm}$ и $\hat{T}_{0}=261 \mathrm{~K}$ с нерасчетностью в диапазоне $50 \leq n \leq 300$, сверхзвуковым потоком с параметрами: $\operatorname{Pr}=0.72$, $\operatorname{Re}=1.87 \cdot 10^{6}, M_{\infty}=4, \hat{T}_{\infty}=70.3 \mathrm{~K}$ в канале длиной $27.69 \mathrm{~cm}$, высотой $11.43 \mathrm{~cm}$ и шириной $15.24 \mathrm{~cm}$ соответственно. Безразмерные параметры вычислительной области следующие: $H_{x}=58, H_{y}=32, H_{z}=24$, где расстояние от входной границы до центра вдуваемой струи составляет $x_{0}=16, y_{0}=16$ калибров. При этом высота пограничного слоя на входе $\delta_{1}=1.65$, где для этой высоты используется 22-26 узлов расчетной сетки. Диапазон рассматриваемого параметра нерасчетности варьировал в пределах $50 \leq n \leq 300$. В работе [13] на примере решения пространственной задачи поперечного вдува звуковой струи с умеренно большими параметрами нерасчетности приведен подробный анализ сходимости численного решения по предлагаемой методике. В соответствии с этим в настоящей работе расчетная сетка была выбрана размером $251 \times 121 \times 151$.

Ниже на рис. 2-6, продемонстрированы результаты численного моделирования с параметром нерасчетности $n=300$. Представленная на рис. 2, $a$ картина поля вектора скорости показывает, что здесь аналогично умеренно большим нерасчетностям формируются два противоположно вращающихся вихря 4 и 5 перед струей, возникающих в результате отрыва набегающего потока, вызванного $\lambda$-образной системой скачков уплотнения. Картина проекции линий тока на плоскость, нормальную к оси $y$ в плоскости симметрии $(y=16)$, хорошо демонстрирует эти вихри (рис. 2, b). На рис. 2, с представлены изобары 

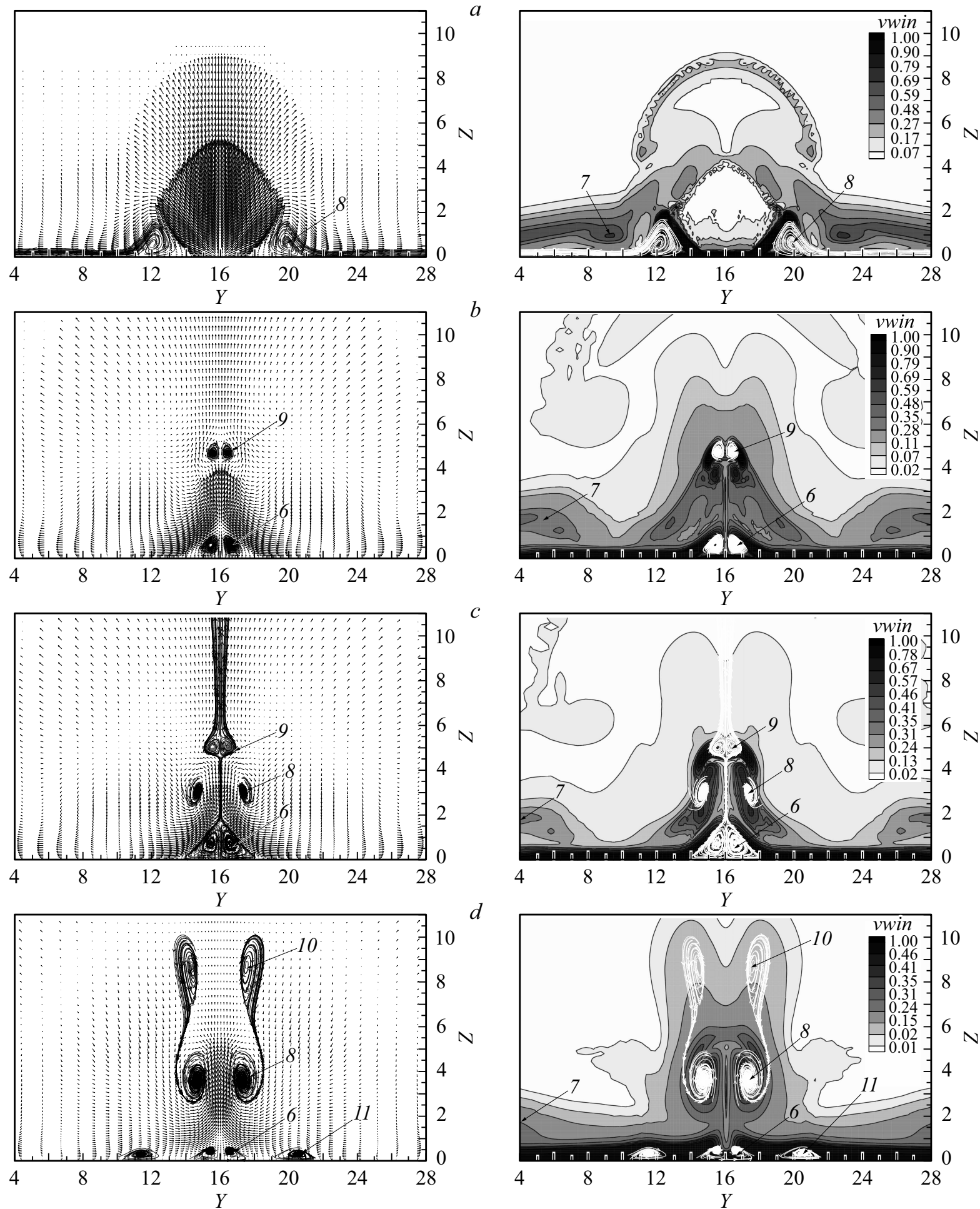

Рис. 4. Поле вектора скорости и проекции линий тока на плоскость, нормальную к оси $x$ (слева), величина завихренности (справа) при $\operatorname{Re}=1.87 \cdot 10^{6}, \operatorname{Pr}=0.72, n=300, M_{0}=1, M_{\infty}=4$ в плоскости $z y: a-$ сечение $x=17, b-x=26.30, c-x=28.18$, $d-x=42.32$.

$\lambda$-образной системы ударных волн (головной, косой и замыкающий скачки уплотнения).

На рис. 2, $a$ также приведен график коэффициента давления на стенке $C_{P}$ (сплошная линия), из которого следует, что по мере приближения к области вдува давление увеличивается, затем его рост замедляется таким образом, что при этом образуется небольшое плато (область I). Далее видно, что давление уменьшается (область II), при этом из поля вектора скорости следует, что область давления II соответствует ядру 


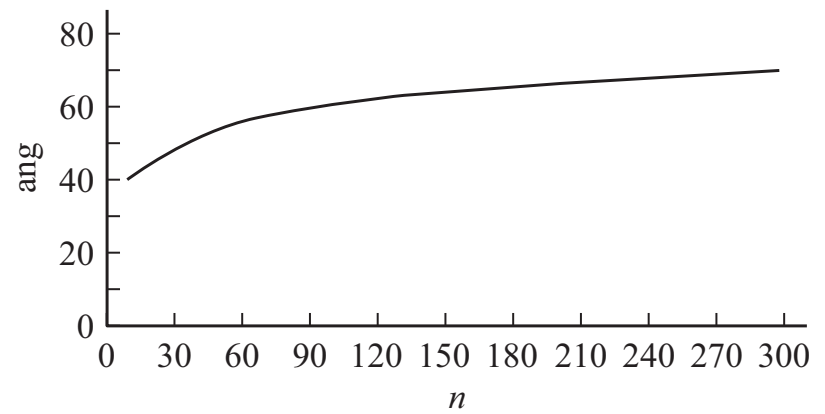

Рис. 5. Влияние параметра нерасчетности на угол наклона головного скачка уплотнения.

подковообразного вихря 4, а его минимальное значение приходится на зону центра вращения. За областью понижения следует резкое возрастание коэффициента давления (область III), где он достигает своего максимального значения (область IV). Вычислительные расчеты показывают, что максимум давления является зоной разделения вращающихся вихрей 4 и 5 (рис. 2,a). Соответствие максимума давления зоне разделения вихрей обусловлено тем, что поток воздуха, проходя участки $\lambda$-образной системы скачков уплотнения, в частности замыкающего скачка, за которым давление достигает наибольшего значения, поворачивает вниз к стенке и проникает в зону отрывного течения, затем растекается в противоположных направлениях с образованием двух вихрей, причем вихрь 4 движется по часовой стрелке, а 5 - против. На графиках распределения изобар (рис. $2, b)$ видно, что максимум давления находится за нижней частью замыкающего скачка уплотнения (значение давления 1.5). График 2, $d$ демонстрирует распределения величины завихренности $\omega=|\operatorname{rot}(\mathbf{u})|$, где отчетливо видна бочкообразная структура, возникающая при вдуве недорасширенной струи в сверхзвуковой поток, так же на рисунке можно наблюдать высокие значения завихренности в области присутствия вышеописанных вихрей.

Из графика рис. 3, $a$ слева (поле вектора скорости и коэффициент давления на стенке $C_{P}$ в плоскости $x z$, $y=18.41)$ можно наблюдать боковое растекание вихрей 4 и 5. Из рисунка видно, что оба вихря разделены максимальным значением давления на графике $C_{P}$ (область IV). Из рисунка следует нечеткая вихревая структура 5 (рис. 3, а справа - проекции линий тока на плоскость, нормальную к оси $X$ ), заметно лишь ее увеличение восходящим потоком. По всей видимости здесь происходит перестройка ориентации центра вращения вихря 5 .

По мере удаления от линии симметрии вихри 4 и 5 существенно перестраиваются. Начиная с сечения $y=18.74$, просматривается только один вихрь 4. Затем его ядро разделяется на два (рис. $3, b$, в плоскости $x z$, $y=20.97)$. Здесь, как следует из рисунка, максимальное значение $C_{P}$ находится не в зоне разделения двух вихрей, что позволяет предполагать о раздвоении вихря 4. Разделенный вихрь быстро распадается, о чем свидетельствует рис. 3, $c$, в плоскости $x z \quad(y=22.98)$, где присутствует только один вихрь. Далее начиная с сечения $y=24.25$, вихревая структура 4 уже не просматривается, по-видимому, здесь также происходит перестройка ориентации центра вращения и его вытягивание в направлении поперечного потока.

На рис. 4, $a$ в плоскости $y z$ (сечение $x=17$ ) показана известная из расчетов при умеренных нерасчетностях [13] пара вихрей (8), которая генерируется вихрем 5 в результате его бокового перетекания. На рисунке слева видно, что величина завихренности $\omega=|\operatorname{rot}(\mathbf{u})|$ в области присутствия вихря 8 имеет большие значения. Как следует из вычислительных экспериментов, для рассматриваемого случая эта пара вихрей обладает, так же как и для умеренных $n=50$ [13], достаточно большой энергией, что позволяет им не только перемещаться далеко вниз по потоку, но и увеличиваться в размерах. Из численных результатов следует, что максимальные размеры бокового вихря здесь наблюдаются в сечении $x=22.85$. Аналогично работе [13] увеличение размеров этих вихрей, по-видимому, обусловлено их локализацией в пристенной области, а рост пограничного слоя проводит к тому, что эти вихри также растут. Из численных расчетов следует, что, начиная с этого сечения, наблюдается уменьшение интенсивности боковых вихрей 8 , а также конец бочкообразной структуры в струе. По мере продвижения вихря 8 вниз по потоку заметна потеря его интенсивности в поперечном направлении, поэтому он не наблюдается в сечениях от $x=23.24$ до $x=26.90$.

Ниже на рис. $4, b$ в плоскости $y z(x=26.30)$ приведена вихревая пара 9, которая формируется у основания диска Маха в результате взаимодействия струи и восходящего потока под струей. Здесь наблюдается вихревой след 6 вблизи стенки в области низкого давления. Величина завихренности на графике в области вихрей 9 и 6 также имеет большие значения. Наличие этих вихревых пар и механизмов их формирования изучено в работе [13] для умеренных параметров нерасчетности в диапазоне $10 \leq n \leq 50$, а также в работе [9] для $n=282$. Таким образом, вышеописанная вихревая система является достаточно устойчивой. Распределение величины завихренности показывает присутствие подковообразного вихря 7 в зоне умеренно больших значений завихренности, локализованных в области, примыкающей к стенке.

Картина, где вновь интенсифицированный вихрь 8 вращается в паре с вихрем 9, представлена на рис. 4, в сечении $x=28.18$. Следует отметить, что для параметра нерасчетности $n=282$ авторы [9] получили эту структуру с двумя вихрями в сечении $x=31$, тогда как численные результаты для этой же нерасчетности выявили это в сечении $x=26.90$. Наблюдается качественное совпадение полученных данных, при этом количественное расхождение можно объяснить различной постановкой входных граничных условий для $x$ и $\omega$ в 

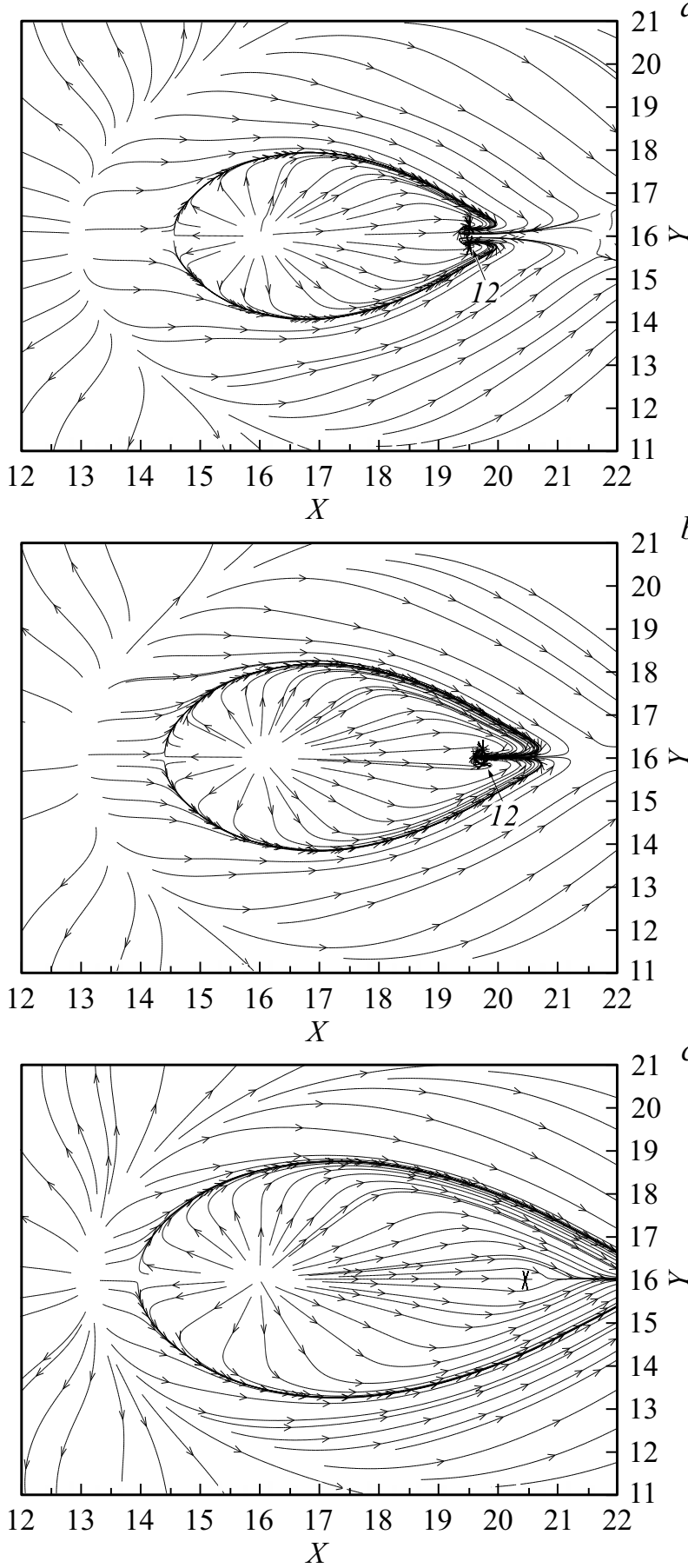

Pис. 6. Проекции линий тока на плоскость, нормальную к оси $z$ (слева), величина завихренности (справа) при Re $=1.87 \cdot 10^{6}$, $\operatorname{Pr}=0.72, n=300, M_{0}=1, M_{\infty}=4$ в плоскости $x y: a-$ сечение $z=0.1235, b-z=0.2648, c-z=0.5958$.

модели турбулентности. Здесь же на графике заметно увеличение размеров вихревой пары 6 .

Далее начиная с сечения $x=30.05$, вихрь 9 увлекается вихрем 8. Об этом свидетельствует тот факт, что направление вращения сформированного вихря совпадает с вращением 8 и сохраняется вплоть до конца расчетной области.

На рис. $4, d$ в плоскости $z y(x=42.32)$ подтверждено присутствие вихревой пары 10 , впервые численно выяв-

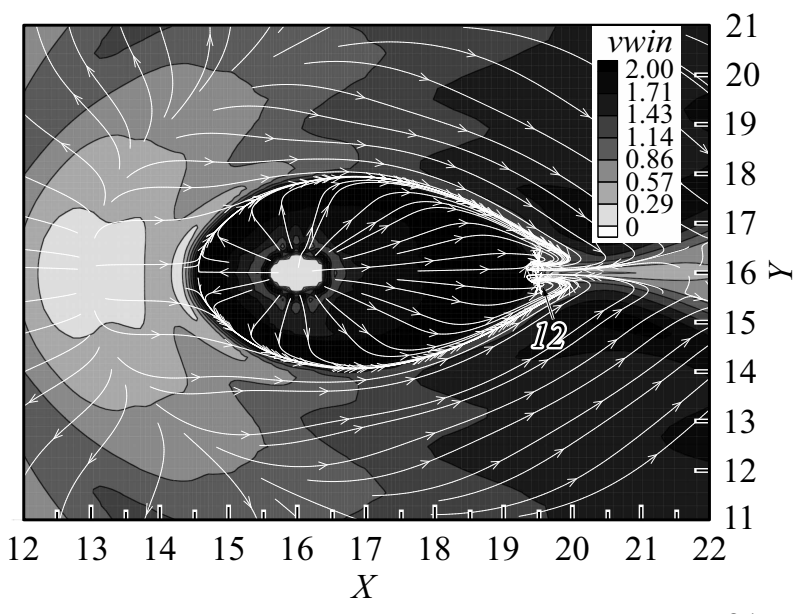

$b$
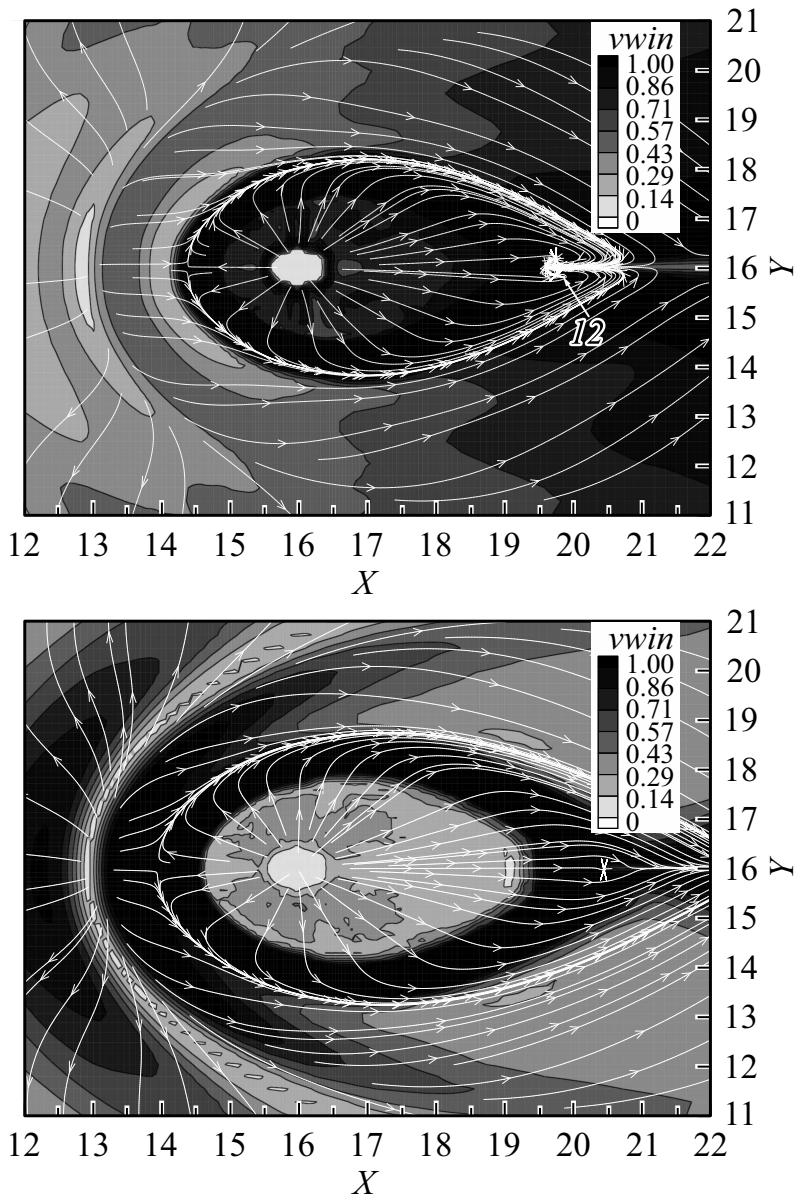

ленной для $n=282$ в [9], а также в [13] для умеренных значений нерасчетности $(n=50)$. Как известно, этот вихрь формируется в результате взаимодействия струи и высокоскоростного натекающего потока, проходящего над вдуваемой струей, и возникает при нерасчетностях выше 20.

Этот же рисунок демонстрирует картину возникновения вихря 11, образованного в результате прилипания струи к плоскости пластины и подробно описанного 
в [13] и, как следует из рисунка, направление его вращения совпадает с вращением следа 6. Численные эксперименты показывают, что в дальнейшем вихри 11 и 6 сливаются, и вниз по потоку наблюдается только один вихревой след. Из картины распределения завихренности видно, что подковообразный вихрь 7 в области умеренных значений отходит от линии симметрии на большое расстояние.

Следует отметить, что локализация вихря 10 по высоте напрямую зависит от интенсивности вдува струи и ударно-волновой структуры. Так, при умеренных значениях нерасчетности (см. работу [13] при $n=50$ ), данный вихрь формируется на высоте от 4 до 5 калибров. В рассматриваемом случае локализация его центра вращения оказалась на уровне $z=8.7$, а при нерасчетности $n=282$ - на высоте $z=8,5$. Так как вихрь (10) расположен между вдуваемой струей и головным скачком уплотнения, можно сделать вывод, что его высота напрямую зависит от угла наклона головной ударной волны. На рис. 5 показан график влияния параметра нерасчетности на головной скачок уплотнения, где ось абсцисс соответствует нерасчетности, ось ординат - углу наклона головного скачка, вычисленного по формуле

$$
\operatorname{ang}=\operatorname{arctg}\left(\max \frac{w}{u}\right) .
$$

Здесь видно, что угол наклона головного скачка уплотнения возрастает с увеличением отношения давления в струе и потоке. Как видно из графика, угол наклона при малых и умеренных значениях нерасчетности резко возрастает, однако, начиная с $n=150$, значения становятся практически постоянными, и высота локализации вихрей 10 будет меняться незначительно, т. е. начиная с $n=150$, высота этих вихрей практически постоянна.

На рис. 6, $a$ в плоскости $x y(z=0.1235)$ можно отчетливо увидеть вихри 12, которые сформированы вблизи стенки в области за струей на небольшом расстоянии от отверстия (в сечении $x=19.5$ ). Данные вихри представлены в работе [10] и носят название horn (роговые вихри). Физический механизм их возникновения состоит в том, что струя, истекая непосредственно из отверстия, взаимодействует с внешним потоком, который, обтекая струю, стремится замкнуться на линии симметрии, что приводит к закрутке встречных потоков в форме двух рогообразных структур. Численные результаты показывают, что этот вихрь формируется и при параметре нерасчетности $n=5$, что свидетельствует об устойчивости данной вихревой системы. Численные результаты показывают, что в сечении $z=0.2648$ (рис. $6, b$ ) вихри замыкаются на линии симметрии, а уже при $z=0.5958$ (рис. 6, c) роговые вихри не просматриваются.

На рис. 7 представлено сравнение численного результата коэффициента давления $C_{P}$ на стенке в плоскости симметрии с данными работы [9]. Как видно из рисунка, получено удовлетворительное согласование численных результатов и экспериментальных данных. Небольшое

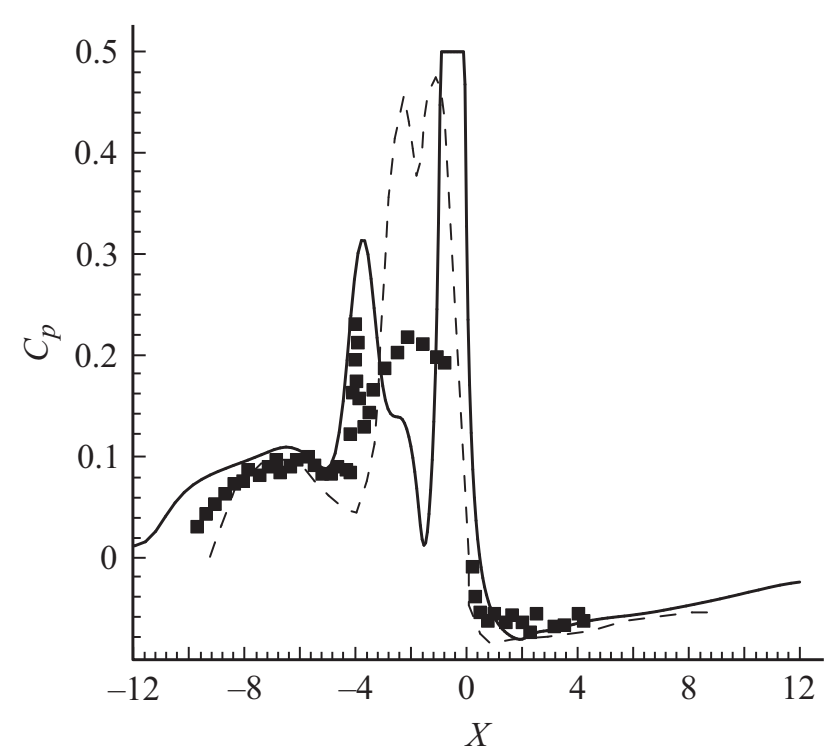

Рис. 7. Распределение коэффициента давления $C_{P}$ на стенке на оси симметрии при $\operatorname{Re}=1.87 \cdot 10^{6}, \operatorname{Pr}=0.72, n=282$, $M_{0}=1, M_{\infty}=4$ : сплошная линия - численные результаты, 口曰 - эксперимент [9], - - - - результаты работы [9].

расхождение расчетных и экспериментальных данных в диапазоне $-4<x<-1$, по всей видимости, обусловлено использованием $k-\omega$ модели турбулентности, которое, возможно, повлияло на область стыковки вдуваемой струи и основного потока вблизи стенки, что свидетельствует о том, что необходима дальнейшая модификация данной модели. Этот факт также подтверждает работа [14], которая посвящена струйному взаимодействию. В настоящей работе изучена применимость различных моделей турбулентности, а именно модели Спаларта-Альмараса, $k-\varepsilon, k-\omega$ и SST-моделей при решении подобных задач на основе сравнения вычислительных и экспериментальных данных. Здесь показано, что использование различных моделей турбулентности приводит к различным численным результатам по распределению давления на стенке перед вдуваемой струей. В частности, расчет с $k-\omega$ моделью турбулентности приводит к занижению результатов численного моделирования по сравнению с экспериментом также в области, близкой к вдуваемой струе.

\section{Заключение}

Проведен детально-сравнительный анализ механизмов образования вихревых структур в результате взаимодействия набегающего потока с вдуваемой струей при умеренных и больших параметрах нерасчетности $50 \leq n \leq 300$. В результате выявлено, что, начиная с $n=50$, происходит перестройка ориентации центров вращения вихрей 4 и 5 в направлении поперечного потока, следствием которой является затруднительная их визуализация в сечениях, поперечных натекающему 
потоку, а также разделение подковообразного вихря 4 на две независимые вихревые структуры по мере удаления его от линии симметрии. Подтверждено наличие роговых вихрей вблизи стенки в области за струей в диапазоне нерасчетностей $10 \leq n \leq 300$, возникающих в результате взаимодействия истекающей струи с внешним потоком, приводящего к закрутке встречных потоков за струей в форме двух рогообразных структур. В ходе построения обобщающей зависимости угла наклона головного скачка уплотнения от параметра нерасчетности показан умеренно линейный рост угла при больших значениях нерасчетности.

\section{Финансирование работы}

Работа выполнена при поддержке Министерства образования и науки Республики Казахстан в рамках грантового финансирования научных исследований по теме: „Численное моделирование пространственных турбулентных сжамиемых течений с вдувом струей и твердых частиц“, 2018-2020 гг., ИРН проекта АР05131555.

\section{Конфликт интересов}

Авторы заявляют, что у них нет конфликта интересов.

\section{Список литературы}

[1] Глаголев А.И., Зубков А.И., Панов Ю.А. // Известия АН СССР. Механика жидкости и газа. 1967. № 3. С. $97-$ 102.

[2] Spaid F.W., Zukoski E.E. // AIAA J. 1968. Vol. 6. N 2. P. 205212.

[3] Schetz J.A. // J. Spacec. Rockets. 1970. Vol. 7. N 2. P. 143149.

[4] Aso S., Inoue K., Yamaguchi K., Tani Y.A // Acta Astronautica. 2009. Vol. 65. N 5, 6. P. 687-695.

[5] Van Lerberghe W.M., Santiago J.G., Dutton J.C., Lucht R.P. // AIAA Journal. 2000. Vol. 38. N 3. P. 470-479.

[6] Chenault C.F., Beran P.S. // AIAA J. 1999. Vol. 37. N 10. P.1257-1269.

[7] Sun De-chaun, HU Chun-bo, CAI Ti-min // Appl. Mathemat. Mechan. 2002. Vol. 23. N 1.

[8] Khali E.H., Yao Y. Mixing flow characteristics for a transverse sonic jet injecting into a supersonic crossflow. In: $53^{\text {rd }}$ AIAA Aerospace Sciences Meeting: AIAA 2015 Sci-Tech Conference, Kissimmee, Florida, USA, 5-9 January 2015.

[9] Viti V., Neel R., Schetz J. // Phys. Fluid. 2009. Vol. 21. P. 1-16.

[10] Lu F.K., Dickmann D.A. Topology of supersonic jet interaction flowfields at high pressure rasio // Presented at the July 1-4, 2008 FLUVISU12-12 ${ }^{\text {th }}$ French Congress on Visualization in Fluid Mechanics, Nice, France.

[11] Бекетаева А.О., Найманова А.Ж. // Прикладная механика и техническая физика. 2011. Т. 52. № 6. C. 1-10. [Beketaeva A.O., Naimanova A.Zh. // J. Appl. Mechan. Tech. Phys. 2011. Vol. 52. N 6. P. 896-904. DOI: $10.1134 / \mathrm{S} 0021894411060071]$

[12] Wei Huang, Jian-guo Tan, Jun Liu, Li Yan // Acta Astronautica. 2015. N 117. P. 142-152.
[13] Бекетаева А.О., Бруель П., Найманова А.Ж. // Прикладная механика и техническая физика. 2015. Т. 56. № 5. C. 50-63. [Beketaeva A.O., Bruel P., Naimanova A.Zh. // J. Appl. Mechan. Tech. Phys. 2015. Vol. 56. N 5. P. 777-788. DOI: $10.1134 / \mathrm{S} 0021894415050041]$

[14] Волков К.Н., Емельянов В.Н., Яковчук М.С. // Прикладная механика и техническая физика. 2015. Т. 56. № 5. Т. 64 75. [Volkov K.N., Emelyanov V.N., Yakovchuk M.C. // J.Appl. Mechan. Techn. Phys. 2015. Vol. 56. N 5. P. 789-798. DOI: $10.1134 / \mathrm{S} 0021894415050053]$

[15] Wilcox D.C. A two-equation turbulence model for wallbounded and free-share flow // AIAA paper. 1993. 93-2905

[16] Poinsot T.J., Lele S.K. // J.Computat. Phys. 1992. N 101. P. 104-129.

[17] Бекетаева А.О., Найманова А.Ж. // Вычислительные технологии. 2007. Т. 12. № 4. С. 17-25.

[18] Bruel P., Naimanova A.Zh. // Thermophys. Aeromechanics. 2010. Vol. 17. N 4. P. 531-541. 